\title{
Creating, implementing, and sustaining an advanced optical spectroscopy laboratory course
}

\author{
Jennifer Blue, S. Burcin Bayram, and S. Douglas Marcum \\ Department of Physics, Miami University, Oxford, Ohio 45056
}

(Received 15 September 2009; accepted 1 February 2010)

\begin{abstract}
An upper-division laboratory course in atomic and molecular spectroscopy is described. Examples of outcomes that also benefit second-year physics laboratories and demonstrations in introductory courses are presented. The overarching goal that drove the development of the course was to assist students in understanding the fundamental connections between atomic and molecular spectra and the underlying structures. A selection of laboratory experiences supporting this goal, and the equipment and techniques necessary to provide them, are outlined. () 2010 American Association of Physics Teachers.
\end{abstract}

[DOI: $10.1119 / 1.3327859]$

\section{INTRODUCTION}

The purpose of this paper is to describe an advanced undergraduate laboratory course that our department has offered in various forms since the mid-1980s. In addition to the standard year of laboratory courses that is part of the introductory physics sequence, ${ }^{1-3}$ physics majors at Miami University are required to complete three laboratory courses during their sophomore year: One each on contemporary physics, electronics, computer interfacing and control, ${ }^{4-6}$ and computational physics. We also offer four advanced laboratory courses to upper-level physics majors and graduate students $^{7,8}$ on digital electronics, materials science, ${ }^{9,10}$ modern optics and laser physics, ${ }^{11}$ and atomic and molecular spectroscopy. ${ }^{12-14}$

The spectroscopy laboratory makes a unique contribution to our curriculum. Students observe atomic and molecular spectra and are then challenged to understand how the spectra relate to the structures of the associated atoms or molecules. A common student reaction to the course is that "it makes quantum mechanics real," a statement that indicates that our goal has been attained.

The origins of both the modern optics and laser physics laboratory, and the spectroscopy course that is the subject of this paper, was an advanced undergraduate elective on spectroscopy and physical optics, which had no laboratory-based component. The addition of a laboratory component began in 1984 when one of us taught this course using existing equipment and borrowed time on research equipment. With the addition of new faculty, collaboration on proposals to fund advanced laboratories was initiated. The two new laboratory courses on optics and spectroscopy were seminally funded by two NSF Instrumentation and Laboratory Improvement grants, with matching funds from the university totaling to $\$ 124,000$. The first grant largely funded the creation of the two new courses, and the second was for enhancement of these successful efforts.

The initial grant and matching funds were used to purchase expensive equipment, most of which served as central equipment items for both courses, including an $\mathrm{Ar}^{+}$laser (5 $\mathrm{W}, \mathrm{cw}$ ) an associated dye laser, a CCD camera/detector used for imaging and spectroscopic detection, a scanning broadband Fabry-Pérot interferometer, an arc-discharge power supply, a variety of arc discharge tubes (Osram lamps), a three-grating spectrometer, optical tables, and several quality lens kits. The second equipment grant was largely devoted to new efforts in both courses to implement diode laser sources and associated new laboratories. In each case we were fortunate to leverage additional funds from the university to purchase associated items such as lenses and mirror mounts, mirrors, and optical fibers, all of which are necessary but difficult to fund externally. Such contributions totaled roughly to $\$ 25,000$, not including significant departmental contributions via machine-shop stock and the time our instrument maker devoted to fabrication of posts and tie-downs for directing light from source to detector. Many undergraduates learned basic machine-shop skills, making such items in an elective course. Periodic institutional awards of equipment funds devoted to undergraduate instruction for both enhancement and replacement of laboratory equipment have also benefited our efforts.

The initial offerings of the new spectroscopy course consisted of general instruction in atomic and molecular physics, coupled with student projects aimed at developing the proposed experiments, and other experiments that developed from faculty research interests, student initiatives and, occasionally, serendipity. In addition to the formal course, many undergraduate independent study hours and a number of graduate student thesis topics were devoted to developing and refining laboratory experiences for the course.

\section{SPECTROSCOPY}

We first give a brief description of the introductory discussion sessions of the course because their content is pedagogically important and informed the basic approach that guided the design of the laboratory experiences. ${ }^{15}$ Blackbody and atomic and molecular spectra have been the subjects of scientific curiosity since Newton. ${ }^{16}$ For example, Newton used a large circular aperture (1/8 in.) drilled in the shade over his bedroom window, the Sun as the light source, and his eye to observe the solar spectrum.

In 1802, Wollaston reported the existence of seven dark lines in the spectrum of sunlight. He passed sunlight through a very narrow slit $(\sim 0.05$ in.) prior to impinging on the prism, and also used eye detection. Between 1814 and 1823, Fraunhofer mapped the solar spectrum including 574 dark (absorption) lines, the seven most prominent being labeled A-G, as well as the underlying blackbody continuum due to the Sun's surface temperature. Observations dating to Fraunhofer helped form the basis for the Planck radiation formula and the subsequent conclusions of Niels Bohr on the ob- 
served spectrum of the hydrogen atom. For nearly half the time that blackbody and atomic and molecular spectra have been known, the study of the optical spectra associated with solids and atoms and molecules in the gas (or plasma) phase was mostly empirical. Nevertheless, even the earliest spectral observations acted as a general guide to developing our course; that is, we employed robust light sources and arranged for sufficient resolution and optical detection sensitivity.

Bohr's results suggested that a single (principal) quantum number is sufficient to account for the various observed series of spectral lines of the $\mathrm{H}$ atom. The beauty and simplicity of this series belies the elegant complexity of the underlying structure. The hidden complexity is revealed by the theoretical contributions of de Broglie and Schrödinger, and the discovery of electron spin by Uhlenbeck and Goudsmit.

When applied to the stationary states of the $\mathrm{H}$ atom, the full quantum mechanical treatment reveals two other quantum numbers in addition to Bohr's principal quantum number $n$. The electronic orbital angular momentum quantum number $l$ and the magnetic quantum number $m$ (which quantizes the $z$-component of the electron's orbital angular momentum), together with the restrictions placed on them implies $n^{2}$ different wave functions, which describe $n^{2}$ different possible states for the electron for each principal quantum number $n$. This degeneracy complicates the energy level diagram for the electronic states of the hydrogen atom over the simple case suggested by Bohr. The discovery of electron spin added another factor of 2 to the complexity of the structure of the simplest of all atoms.

The result is that four quantum numbers $(n, l, m$, and $s)$ are required to specify a given stationary state of an electron bound to a proton. This set of quantum indices, coupled with the weak statement of the Pauli exclusion principle and its generalization to other atoms, provides the foundation for the Periodic Table of the elements. The challenge is to understand why the spectrum of atomic hydrogen is so simple and then to search for reasons that the presence of more than one electron significantly increases the complexity of the spectra of other atoms. The inclusion of the effect of nonzero net nuclear spin completes the quantum structure of atoms. The spectra of simple diatomic molecules are further complicated by the addition of quantized vibrational and rotational motions. The effects of those additional degrees of freedom on the observed spectrum of the homonuclear diatomic molecule $\mathrm{N}_{2}$ comprises the introduction to molecular structure contained in the course. A guided discovery approach is used throughout.

\section{THE COURSE}

Spectroscopy of Atoms and Molecules is a one-semester, four credit course that emphasizes experimental techniques found in research. The course typically meets three times a week, with two 1-h classroom meetings and one 3-h laboratory meeting. We use Herzberg's classic Atomic Spectra and Atomic Structure ${ }^{17}$ as a textbook. Texts on reserve in the department form important additional student resources, especially Haken and Wolf's The Physics of Atoms and Quanta, ${ }^{18}$ Svanberg's Atomic and Molecular Spectroscopy ${ }^{19}$ and Herzberg's Molecular Spectra and Molecular Structure I: Spectra of Diatomic Molecules. ${ }^{20}$ Many handouts and the individual laboratory handouts have been developed over the years.
After the completion of each formal experiment, students are required to submit a written report in the style of a professional paper. Student assessment is based primarily on the laboratory reports, with a small portion derived from performance on periodic quizzes. The experiments are complemented by classroom and other laboratory experiences, which include group discussions, a variety of physical phenomena explored via demonstrations, and oral presentations by individual students.

\section{LABORATORY EXPERIENCES}

\section{A. Introduction to blackbody, atomic, and molecular spectra}

The initial laboratory experience uses a circa $192090^{\circ}$ constant-deviation prism spectrometer and eye detection. Light sources include a clear, linear-filament incandescent bulb powered by a variac, AC capillary discharge tubes filled with hydrogen, water vapor, helium, mercury, and nitrogen. These various light sources are inexpensive and durable. The spectrometer has all the essential attributes of any optical spectrometer: Entrance slit (variable width), dispersing element (the prism), focusing elements (a telescope), and detection apparatus (the student's eyes and an optional film plate camera which allows spectra to be recorded). By using such an instrument (even a hand-held plastic transmission grating) and the available sources, students can measure blackbody spectra at a variety of temperatures, the spectra of the two simplest atoms ( $\mathrm{H}$ and $\mathrm{He})$, observe that a capillary discharge in water vapor primarily yields the Balmer lines, view the spectrum of a heavy element that is commonly found in classrooms and commercial buildings (mercury-vapor lamps), and gain an appreciation for the complexity of molecular spectra.

Important experimental considerations such as the relative spectral response of spectroscopy systems can be easily addressed by investigating the various optical components and considering, most importantly in this case, the spectral sensitivity of the detector (the human eye). It is easy to argue that the average electron energy in AC capillary discharge tubes is small compared to the separation between the ground and first excited states of atomic hydrogen. Thus, the Boltzmann factor results in decreasing excited state population with increasing $n$. Asking students to compare the brightness of the Balmer- $\alpha$ and $\beta$ lines almost always results in the consensus opinion that they appear to be roughly the same intensity. The population argument based on the Boltzmann factor suggests that $\mathrm{H}-\alpha(n=3-2)$ should be brighter than $\mathrm{H}-\beta(n=4-2)$. The conclusion is that their eyes must be more sensitive in the blue-green region than in the red. The observation of the $\mathrm{H}$-atom spectrum with CCD-array detectors during the first formal laboratory experiment shows a more realistic intensity distribution for the Balmer lines. The important discovery is that the relative spectral response of the entire spectroscopic system needs to be known so that the appropriate corrections to observed spectra are made if necessary.

The applicability of a blackbody source at a known temperature as a tool for relative spectral response calibration of a spectroscopy system can be easily motivated with the same simple instruments. Visual comparison of the spectra of hydrogen and helium provides a convincing argument that the addition of a second electron enormously complicates the 
emission spectrum. The observation of a discharge in molecular nitrogen emphasizes that molecular spectra are even more complex. In this case, the effect of the entrance slit width is striking, demonstrating both the concept of spectral resolution and the reason molecular spectra are said to consist of bands. The results are qualitative, and no formal laboratory report is required.

\section{B. The spectra of atomic hydrogen and deuterium}

The first quantitative laboratory experiment for which students are required to write a formal report involves the isotope shift observed in an AC capillary discharge in a mixture of hydrogen and deuterium. A hydrogen/deuterium capillary discharge tube is only slightly more expensive than a pure hydrogen-filled source. By using a CCD camera placed at the focal plane of a spectrograph (acquired from surplus sources), the wavelength shift due to the difference between the finite nuclear mass of a proton and a deuteron can be directly observed. Careful simultaneous measurements of the Balmer lines in the two spectra yields, via Balmer's empirical formula, the small difference in the Rydberg constant for $\mathrm{H}$ and $\mathrm{D}$ and, via the Bohr model, allows the calculation of the difference in the ionization potentials for these isotopes. Very accurate measurements of the spectra of $H$ and $D$ yield decent estimates of the binding energy of the deuteron. The $\mathrm{H}-\mathrm{D}$ isotope shift is the largest possible because it involves the maximum fractional difference in the nuclear mass. Not only does this laboratory show the effect of a purely classical mechanical effect (different reduced masses for the two atomic systems) in a quantum mechanical system, but it also suggests the possibility of laser isotope separation techniques (ionize one isotope, but not the other, and then separate using electric fields).

The relative intensities of the various Balmer lines are apparent in the measured spectra, and students are reminded of the discussion of the energy distribution of the electrons in the discharge mentioned previously. The isotope shift is revisited later in the course when the absorption spectrum of rubidium vapor is measured.

A simplified version of this experiment has been implemented in the sophomore contemporary physics laboratory, which uses less expensive hydrogen capillary discharge tubes, and recently available lower-resolution and much-lessexpensive CCD spectrometer systems (see, for example, fixed grating CCD spectrometers with USB power/inputoutput available from Ocean Optics). The latter approach also allows the direct viewing of low-resolution optical spectra projected on a screen in a typical classroom environment where the number of students depends only on the room capacity. This example illustrates how advanced laboratory developments can be used in a simplified way to benefit introductory courses with a modern physics component, as well as sophomore-level contemporary physics laboratory courses.

\section{The spectrum of helium}

In another simple laboratory experiment the spectrum of an AC capillary discharge in helium is recorded using another scanning monochromator (0.5 m Jarrell-Ash) acquired through surplus sources and photomultiplier (PMT) detection. The details of the spectroscopy system used are unimportant as long as reasonably accurate emission wavelength measurements are possible. The major advantages of using PMT detection are the easily adjustable gain, and the demonstration of the ability to follow the AC time variation of the light source, although the latter capability is not required for the purposes of this laboratory.

By this time in the course, we develop spectroscopic notation (L-S coupling) $n^{2 S+1} L_{J}$, where $n$ is the principal quantum number of the excited electron, $S$ is the total electronic spin angular momentum with multiplicity $2 S+1, L$ is the total electronic orbital angular momentum, and $J$ represents the total electronic angular momenta, $J=L+S$. Prediction of ground and excited state terms has been discussed, and dipole selection rules have been established. The energy level diagram for $\mathrm{He}$ is provided to students along with a table of the observed emission wavelengths and the corresponding (singlet and triplet) electron configurations for the upper and lower levels of each transition wavelength. Students are asked to determine the spectroscopic notation of the levels involved in each observed transition, verify that the dipole selection rules are followed, and to discuss the general character of the term diagram in conjunction with the observed relative intensities of the various recorded transitions.

Positive outcomes of this experiment include students concluding that two term diagrams are necessary to explain the spectrum of He, given that both singlet and triplet excited states are possible once an electron is excited out of the ground $(n=1)$ level; no intercombination (the $\Delta S=0$ rule) lines are observed; the lower the orbital angular momentum of the excited electron, the lower the energy of the term (the $l$-degeneracy in hydrogen is broken); the higher the multiplicity, the lower the energy for a given electron configuration; the various sublevels predicted by L-S coupling are not observable in the He spectrum; and as with the spectrum of the $\mathrm{H}$ atom, the higher the energy level, the lower the electron-impact-excited population. The prediction of longlived (metastable) levels in $\mathrm{He}$ is also motivated. The major atomic-structure-related observations are that the $l$-degeneracy found in the $\mathrm{H}$ spectrum is broken in $\mathrm{He}$, but the spin-orbit interaction is still too weak in helium to show fine structure splitting.

In this way, the simplest example of the addition of electron-electron interactions to an atomic structure is revealed. The effect of the additional electron in the $n=1$ level is to screen the nucleus from the excited electron. The lower the orbital angular moment of the excited electron, the more time it spends in the vicinity of the nucleus, resulting in a stronger interaction with the nucleus (that is, lower $L$ implies more tightly bound). In contrast, high angular momentum states for the excited electron more effectively shield the excited electron from the nucleus, giving less tightly bound levels. Additionally, for high $n$ the one degeneracy is largely recovered. These observations are reinforced by the next laboratory experiment in the course sequence.

\section{Alkali spectra: Fine structure splitting and selection rules for dipole transitions}

The alkali metals, like atomic hydrogen, are one-electron atoms because each has one electron outside closed shells, giving each a total spin of $S=1 / 2$ and a multiplicity of 2 (doublet terms). They represent the simplest analogs of the template hydrogen atom and also represent a sequence of ever-larger nuclear charge $Z$. The brightest lines in each of the alkali spectra are the D lines (after the Fraunhofer lines 


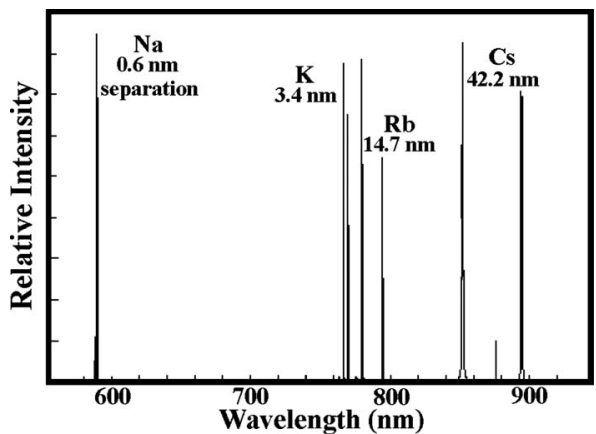

Fig. 1. The alkali D lines. Note the progression toward longer wavelength and the significant increase in spacing for the heavier atoms.

that he originally labeled D). Fraunhofer's highest resolution solar spectra showed that the D line was actually two closely spaced absorption lines, which he labeled $\mathrm{D}_{1}$ and $\mathrm{D}_{2}$.

Light sources used for this experiment are arc discharge tubes (Osram lamps) filled with solid sodium, potassium, rubidium, and cesium and a low density of argon; they provide the various alkali emission spectra without significant broadening. Unfortunately, such lamps are expensive relative to those sources mentioned so far, and all of the heavier alkalis are found to be slightly contaminated with sodium. However, they are robust, having survived many hours of use since their purchase in the early 1990s, and they are bright. They also operate well in strong external magnetic fields, which allows investigation of the magnetic quantum number via the Zeeman effect (see the following).

The CCD camera and three-grating spectrometer work well for this investigation. The spectrometer (Jarrell-Ash Monospec 26) used in the experiment is compact $(0.26 \mathrm{~m})$ with interchangeable fixed entrance slits and three gratings $(150,600$, and 2400 grooves $/ \mathrm{mm})$ mounted on a turret that provides roughly 240,60 , and $15 \mathrm{~nm}$ bands, respectively, to impinge on the $512 \times 512 \mathrm{CCD}$ array. The CCD array is sensitive over the range of 300-1100 $\mathrm{nm}$. The highest resolution grating is necessary to resolve the $\mathrm{Na} \mathrm{D}$ lines, which are separated by only $0.6 \mathrm{~nm}$. K and $\mathrm{Rb} \mathrm{D}$ lines are easily resolvable with the medium resolution configuration, and the lowest resolution grating is satisfactory for the large spinorbit splitting observed in Cs. The D lines are simple to identify given that they are by far the brightest emission lines from each lamp.

The D lines for each of the four alkalis are shown in Fig. 1. Simple wavelength measurements of each pair of D lines, followed by conversion to photon energy and subtraction, yield the spin-orbit interaction energy for electrons in the first excited states of each alkali. Because the energy splitting is given by twice the Bohr magneton multiplied by the mag-

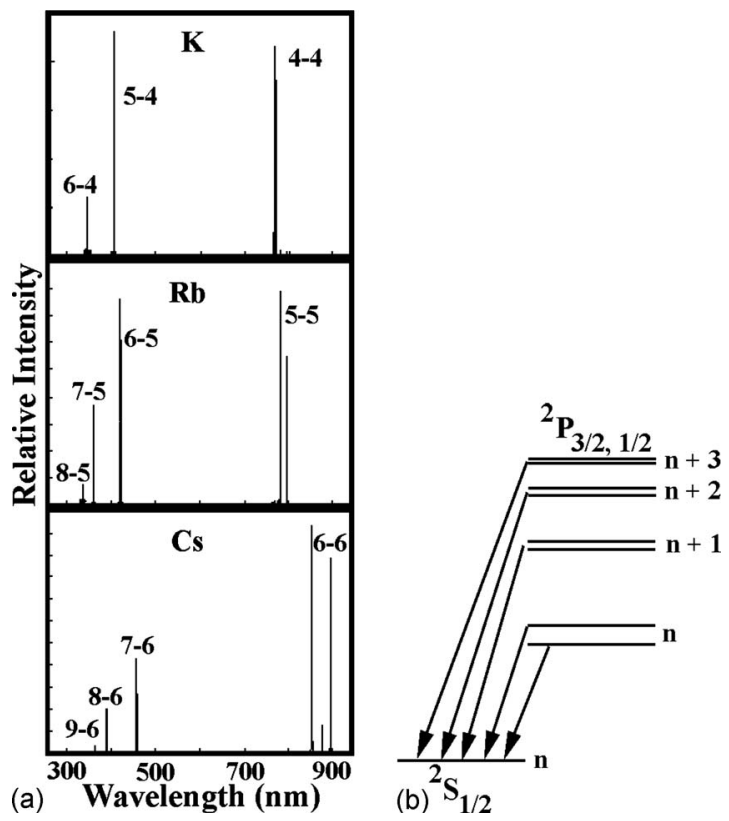

Fig. 2. (a) Alkali principal series showing the decrease in the magnetic field felt by the electron with increasing principal quantum number. (b) The excited state structure that gives rise to the spectra in (a).

netic field due to the orbital motion of the excited electron, the magnetic field experienced by the electron in the first excited state of each alkali is easily calculated. The students are told that the energy splitting is proportional to $Z$ raised to some power divided by the principal quantum number cubed and are asked to discover the $Z$-dependence. Plotting the observed energy splitting versus $Z^{4} / n^{3}$ yields a beautifully straight line. Table I summarizes the relevant experimental results. Note that extrapolation of the observed splitting to $Z=1$ or 2 answers the question why spin-orbit splitting is not easily observed in hydrogen. The $l$-degeneracy in $\mathrm{H}$ is already understood due to the lack of screening in the pure Coulomb potential in the $\mathrm{H}$ atom (or any hydrogenlike ion).

Further results that are easily obtained by identifying the principal series of the three heaviest alkalis (the brightest series emanating from each lamp) are shown in Fig. 2(a). Two observations are apparent: Each series converges to a limit, just as the Balmer series (hydrogenlike). Furthermore, the splitting of the principle series doublets decreases with increasing $n$. The latter is consistent with diminishing magnetic field due to more distant orbital motions. Figure 2(b) shows the principal series portion of an alkali energy level diagram that results from the trends apparent in Fig. 2(a).

Other interesting measurements that are possible during such an experiment include, for example, the $7^{2} D_{J}$ to $6{ }^{2} D_{J^{\prime}}$

Table I. Spin-orbit splitting of the alkali D lines and the measured magnetic fields involved.

\begin{tabular}{lccccc}
\hline \hline Atom $(n)$ & & $\mathrm{Na}(3)$ & $\mathrm{K}(4)$ & $\mathrm{Rb}(5)$ & $\mathrm{Cs}(6)$ \\
\hline Wavelength $(\mathrm{nm})$ & $\mathrm{D}_{1}$ & 589.592 & 769.896 & 794.760 & 894.395 \\
& $\mathrm{D}_{2}$ & 588.995 & 766.490 & 780.023 & 852.124 \\
Energy $(\mathrm{eV})$ & $\mathrm{E}_{1}$ & 2.1031 & 1.6161 & 1.5602 & 1.3864 \\
& $\mathrm{E}_{2}$ & 2.1053 & 1.6178 & 1.5897 & 1.4552 \\
Difference $(\mathrm{eV})$ & & 0.0021 & 0.0072 & 0.0295 & 0.0688 \\
$B(\mathrm{~T})$ & 18 & 62 & 254 & 594 \\
\hline \hline
\end{tabular}




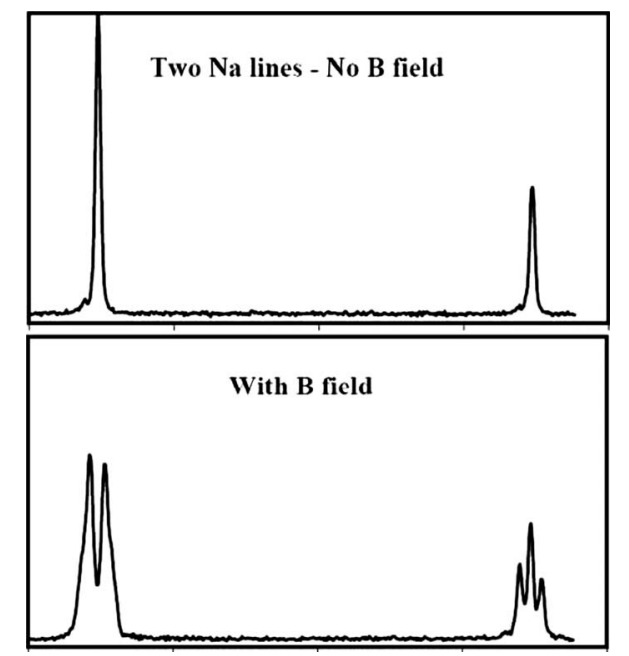

Fig. 3. A pair of sodium emission lines with and without an externally applied field.

transitions in atomic cesium. Such transitions allow the verification of the $\Delta J=0, \pm 1$ dipole selection rule and the prediction of the intensity ratios within doublets (using statistical weights and the sum rule).

\section{E. Zeeman effect in sodium}

The experimental apparatus consists of a sodium discharge tube, a low-cost linear polarizer, a focusing lens coupled to an optical fiber and coupled, in turn, to the entrance slit of a $3 / 4 \mathrm{~m}$ spectrometer, and a CCD array attached at the focal plane of the spectrometer. The spectrometer (Jarrell-Ash) is capable of operating in third order, achieving the resolution necessary to observe Zeeman splitting with the modest field achievable (maximum $5 \mathrm{kG}$, short duration) with the available magnet/power supply. The sodium lamp is positioned between the pole caps of an (uncooled) electromagnet (discarded from our Department of Chemistry) and powered by an old high-current, low voltage DC power supply. The observation of sodium emission lines at right angles to the field without and with an external field applied is shown in Fig. 3.

Sodium exhibits the anomalous Zeeman effect. Full resolution of the Zeeman components (not shown) is possible by inserting a linear polarizer in front of the optical fiber and rotating it by $90^{\circ}$. This experiment offers a direct demonstration of the atomic origins of polarization.

\section{F. Hyperfine structure of rubidium}

The final major component of the atomic structure involves the nuclear analog of spin-orbit splitting. Nuclei can also possess a net spin, but the nuclear magneton is roughly 2000 times smaller than the Bohr magneton, which is important in the splitting of electronic energy levels. The total electronic angular momentum is quantified by the quantum number $J=L+S$, and the total atomic angular momentum is represented by the quantum number $F=I+J$, where $I$ is the total nuclear spin angular momentum and $J$ is the total electronic angular momentum. Given the magnitude of the nuclear magneton relative to that of the Bohr magneton, the spin-orbit splitting due to a net nuclear spin is about 2000 times smaller for hyperfine splitting, which implies that spectral resolution requirements are best met by using very nar-

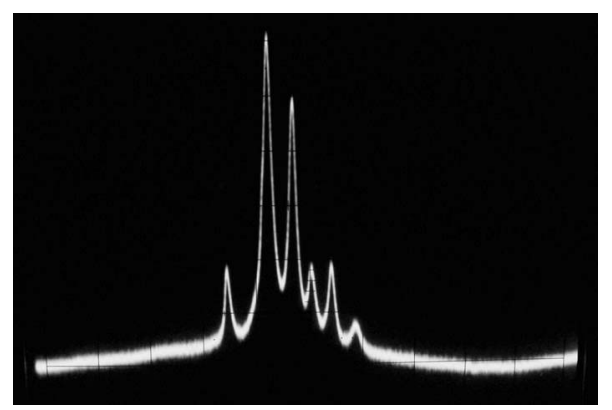

Fig. 4. A sample hyperfine spectrum showing transitions in rubidium-85 from $F=3$ to $F^{\prime}=2,3,4$.

row linewidth lasers as a probe of the hyperfine structure. The hyperfine structure shown in Fig. 4 was obtained using a locally built external cavity tuned diode laser in a standard saturated absorption spectroscopy setup.

\section{G. Raman scattering in liquid nitrogen}

The first molecular spectroscopy experiment also uses a laser light source. ${ }^{21}$ Raman scattering spectroscopy is a powerful technique for determining the internal structure of molecules and is an ideal demonstration of the vibrational transitions of gas and liquid phase molecules and crystals. We designed a straightforward and highly visual experiment for an advanced undergraduate spectroscopy laboratory to demonstrate Raman scattering spectroscopy by measuring the vibrational energy spacing of nitrogen molecules in the liquid phase. We used a pulsed Nd:YAG laser with high peak power, which leads to a plethora of nonlinear optical phenomena. The presence of highly visible stimulated Raman scattering greatly enhances the normal Raman-shifted signal, allowing for a more engaging laboratory experience in comparison to traditional Raman scattering experiments. The technique permits direct visual observation of nonlinear optical effects such as four-wave mixing including coherent Stokes and anti-Stokes Raman scattering, stimulated Raman scattering, and the associated cascaded multiorder stimulated Raman scattering, and thermal blooming. Full details of this experiment are in Ref. 21. The most relevant measurement from the viewpoint of molecular structure is that of the spacing between the ground and first excited vibrational levels in the ground electronic state of $\mathrm{N}_{2}$. The direct demonstration of inelastic scattering of a photon is also an important pedagogical outcome.

\section{H. Vibrational structure of $\mathrm{N}_{2}$}

The goal of this experiment is to assist students in understanding the vibrational structure of diatomic molecule and the deviation of real molecular potentials from the simple harmonic oscillator model. The complexity of typical molecular spectra can be daunting. Our solution is to selectively excite particular electronic bands using DC discharges in noble gas- $\mathrm{N}_{2}$ mixtures, making use of energy pooling in noble gas metastable levels as in the $\mathrm{He}-\mathrm{Ne}$ laser. In the experiment, the selectively excited nitrogen molecules formed in a low pressure DC discharge give emission bands primarily involving a single upper electronic state. Students must first determine the upper and lower vibrational quantum numbers associated with each observed bands and then de- 
termine the energy spacing between these levels. This determination must be informed by the Franck-Condon principle, which governs the intensity distributions in electronic bands. $^{22}$

We use a DC discharge containing roughly $1 \% \mathrm{~N}_{2}$ and an Ar $-\mathrm{N}_{2}$ mixture at a pressure of around 5 Torr. The excitation mechanism is direct electron-impact excitation of the argon atoms. Because argon has metastable states at 11.5 and 11.7 $\mathrm{eV}$, large populations of these metastable states accumulate. These metastable states have energies that are resonant with the $C$ state in neutral $\mathrm{N}_{2}$. Therefore, the efficient population of those electronic levels in nitrogen proceeds by the excitation exchange reaction,

$$
\operatorname{Ar}^{*}(M)+\mathrm{N}_{2}(X, v=0) \rightarrow \mathrm{N}_{2}\left(C, v^{\prime}\right)+\operatorname{Ar},
$$

and is followed by spontaneous emission via

$$
\mathrm{N}_{2}\left(C, v^{\prime}\right) \rightarrow \mathrm{N}_{2}\left(B, v^{\prime \prime}\right)+h \nu .
$$

In these reactions, $v^{\prime}$ varies from 0 to 4 , and $v^{\prime \prime}$ can vary between 0 and 6 or 7 , giving rise to emission bands between about 330 and $440 \mathrm{~nm}$. Thus, the medium resolution configuration of the same spectroscopic system used for the exploration of the alkali spectra can be used to measure the spectrum of electronic transitions from a single upper state to a particular lower electronic state. Because a number of vibrational levels are populated in the upper electronic state, allowed transitions to all possible vibrational levels in the lower electronic state provide, via the resulting emissions, sufficient data to determine the vibrational structure of the lower portions of both potential wells. The student's goal is to quantify deviations from the prediction of the simple harmonic oscillator model for the vibrational level spacing.

After students determine the upper and lower vibrational quantum numbers associated with each observed band, they are asked to construct an energy level diagram showing the resulting vibrational structures and then discuss any deviations from the prediction of equal spacing. We provide students the identities of the upper and lower vibrational levels involved in the formation of the brightest observed band. Students are given the hint that the brightest band connects $v^{\prime}=0$ in the upper electronic state to $v^{\prime \prime}=0$ in the lower electronic state. A typical spectrum is shown in Fig. 5(a), with the resulting energy level diagram appearing in Fig. 5(b). Note that the spacing between vibrational levels decreases slightly as the vibrational quantum number increases, in agreement with the anharmonic nature of molecular potentials.

\section{Rotational structure of $\mathbf{N}_{2}^{+}$}

We focus on the rotational structure of the nitrogen molecular ions. Selective excitation of a particular molecular ion state is accomplished by Penning ionization. Penning ionization occurs when an atomic metastable state (helium in this case) is sufficiently energetic to cause ionization of a collision partner (ground state $\mathrm{N}_{2}$ here). If the ion that is created in that way is in an excited electronic state, the resulting spontaneous emission to a lower electronic state of the ion forms the electronic spectrum that is of interest in this experiment.

The same discharge apparatus used in the previous experiment is used with helium substituting for argon. Helium has metastable levels at 19.8 and $20.6 \mathrm{eV}$, which are resonant with the $\mathrm{N}_{2}^{+}\left(B, v^{\prime}\right)$ state, where the vibrational level $v^{\prime}=0$ is
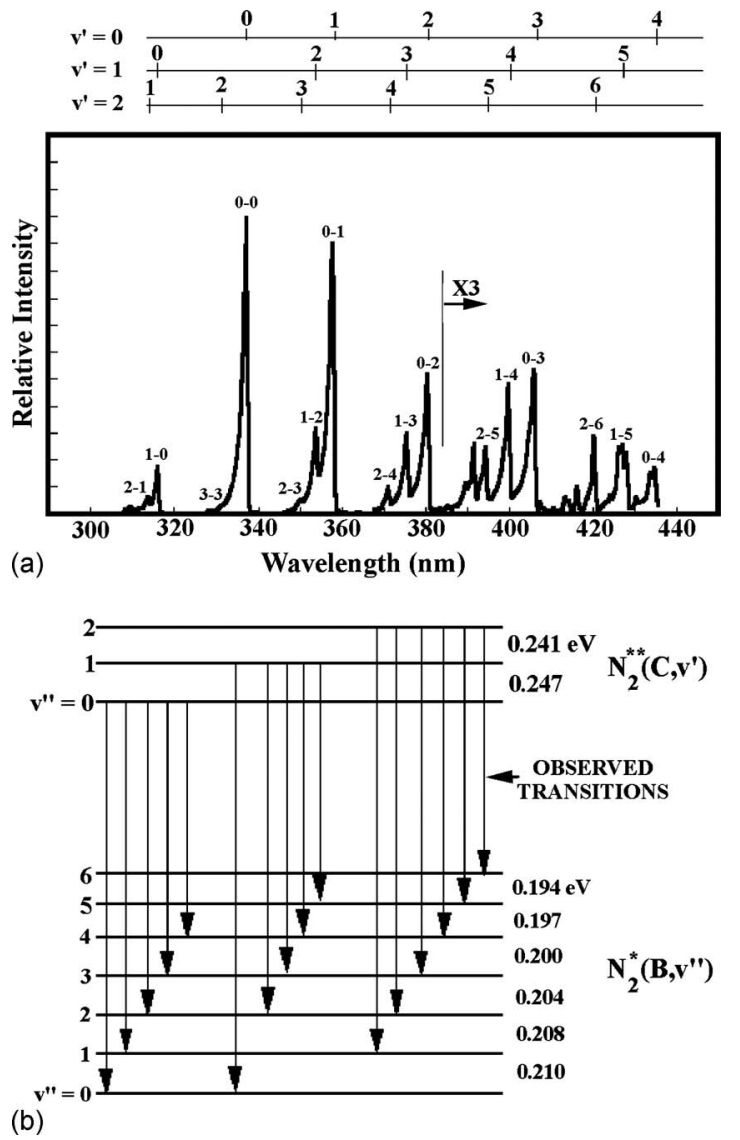

Fig. 5. (a) Observed $\mathrm{N}_{2}\left(C, v^{\prime}\right) \rightarrow \mathrm{N}_{2}\left(B, v^{\prime \prime}\right)$ emission bands. Above $380 \mathrm{~nm}$, the intensity has been multiplied by a factor of 3. (b) Vibrational energy level diagrams derived from the band spectra shown in (a).

most heavily populated by the Penning reaction. The subsequent emission to the ground electronic ion state proceeds by

$$
\mathrm{N}_{2}^{+}\left(B, v^{\prime}=0\right) \rightarrow \mathrm{N}_{2}^{+}\left(X, v^{\prime \prime}=0\right)+h \nu,
$$

forming an emission band near $391.4 \mathrm{~nm}$.

Because molecular rotational energy spacings are small compared to typical vibrational spacings, higher resolution is necessary to resolve the rotational structure associated with any molecular transition. The same spectrometer/detector arrangement used for the Zeeman structure laboratory is sufficient for this task. A typical spectrum obtained in this experiment is shown in Fig. 6. Measurable quantities include the rotational inertia of nitrogen molecular ions. The distribution of intensity over the well-resolved rotational branch is governed by Boltzmann statistics, assuming that the discharge gas is in thermal equilibrium. The resulting rotational inertia (or rotational constant) agrees very well with published values. $^{23}$ Because the lamp operates at near room temperature, a Boltzmann plot yields a temperature of $320 \pm 20 \mathrm{~K}$. The details of extracting the rotational temperature and the rotational constant are given in Ref. 23.

\section{CONCLUSIONS AND COMMENTS}

Our goal as a department, and for the most part our practice, is to continually update the physics content and techniques in our upper-level laboratory courses. Therefore, we try to adapt the advanced laboratory activities to the sophomore-level laboratory course and adapt sophomore- 




Fig. 6. High-resolution emission spectrum near $391.4 \mathrm{~nm}$ resulting from the reaction shown. Note that two helium lines conveniently provide the wavelength calibration necessary to extract measurements from the spectrum.

level activities for the introductory laboratory course. The practice requires periodic infusions of capital from the department and the university to fund additional equipment. Some of the simpler equipment items can be fabricated in our machine shop, but the time of our instrument maker is valuable.

We have found that sustaining our advanced laboratory course in atomic and molecular spectroscopy has resulted in as much work as was its implementation. The course requires a large time commitment from the faculty. Due to staffing limitations, the department offers two of our four advanced laboratory courses each year on an alternating basis so that advanced undergraduates and master's students have an opportunity to take all four courses during a two-year period. The expensive nature of much of our equipment means that we can accommodate only four students in a laboratory section; we cannot afford to buy or make 12 setups for each activity. If the course is popular, we need to offer multiple laboratory sections. In recent years, the course has had as many as 12 students, which has necessitated three 3-h laboratory sessions in addition to the two 1-h discussion sessions. As a result, the professor teaching the course has had 11 contact hours a week, plus setup time, for which he or she gets the same credit as a professor teaching a 4-h lecture course.

Because of our belief in the importance of providing excellent laboratory experiences for our students, we accept the costs of doing so. The efforts needed to obtain both external and internal funding, and the hours of our own time, are well spent to "make quantum mechanics real" for our students.

\section{ACKNOWLEDGMENTS}

The funding for this course was provided, in part, by the National Science Foundation under Grant Nos. USE9051252 and DUE-9452517. Thanks are due to Dr. Perry Rice and Dr. Jan Yarrison-Rice for their work with the original grants. Thanks are also due to Dr. Samir Bali for his contributions to the hyperfine structure laboratory.
${ }^{1}$ Joshua Jacob and Jennifer Blue, "Reform of introductory laboratories: Baseline data," presented at the Winter Meeting of the AAPT, Anchorage, 2006.

${ }^{2}$ Jennifer Blue and Mark Fisher, "Adapting a photoelectric effect lab for the first-year laboratory," presented at the Winter Meeting of the AAPT, Chicago, IL, 2009.

${ }^{3}$ Jennifer Blue, "Evidence of critical thinking in undergraduate laboratory reports," presented at the Summer Meeting of the AAPT, Ann Arbor, MI, 2009.

${ }^{4}$ Jeffrey Clayhold and Joseph Priest, "Automated heat capacity apparatus on a circuit board," Am. J. Phys. 75, 379-381 (2007).

${ }^{5}$ Jeffrey Clayhold and Joseph Priest, "Inexpensive high-precision capacitance measurements and their applications in undergraduate laboratories," Am. J. Phys. 76, 1167-1171 (2008).

${ }^{6}$ Jeffrey Clayhold and Joseph Priest, "Inexpensive precision capacitance measurement and its applications in undergraduate laboratories," presented at the Summer Meeting of the AAPT, Edmonton, 2008.

${ }^{7}$ S. Douglas Marcum, H. Jaeger, and Jan M. Yarrison-Rice, "Advanced undergraduate laboratories at Miami University," presented at the Fall Meeting of the Ohio Section of the APS, Oxford, OH, 1997.

${ }^{8}$ S. Douglas Marcum and Stephen G. Alexander, "The undergraduate physics programs at Miami University-A case study," presented at the AIP/AAPT Conference on Revitalization of Undergraduate Physics Programs, Alexandria, VA, 1998.

${ }^{9}$ Herbert Jaeger, Michael J. Pechan, and Daniel K. Lottis, "Materials physics: A new contemporary physics laboratory,” Am. J. Phys. 66, 724-730 (1998).

${ }^{10}$ Wesley Burgei, Michael J. Pechan, and Herbert Jaeger, "A simple vibrating sample magnetometer for use in a materials physics course," Am. J. Phys. 71, 825-828 (2003).

${ }^{11}$ S. Douglas Marcum, Perry R. Rice, and Jan M. Yarrison-Rice, "Laser physics and non-linear optics undergraduate laboratories at Miami University," presented at the Summer Meeting of the AAPT, Spokane, WA, 1995.

${ }^{12}$ S. Douglas Marcum, Jan M. Yarrison-Rice, and, Perry R. Rice, "Advanced undergraduate atomic and molecular physics laboratory experiments," presented at the Summer meeting of the AAPT, College Park, MD, 1996.

${ }^{13}$ S. Burcin Bayram, "Vibrational spectra of the nitrogen molecules in the liquid and gas phase," presented at the AAPT Topical Conference on Advanced Laboratories, Ann Arbor, MI, 2009.

${ }^{14}$ S. Douglas Marcum, "Creating, implementing, and sustaining an advanced undergraduate laboratory course," presented at the Summer Meeting of the AAPT, Ann Arbor, MI, 2009.

${ }^{15}$ For good treatments of the history of spectroscopy, see Raymond A. Serway, Clement J. Moses, and Curt A. Moyer, Modern Physics (Thompson Brooks/Cole, Belmont, CA, 2005), Chap. 4; or David Cassidy, Gerald Holton, and James Rutherford, Understanding Physics (Springer, New York, 2002), Chap. 14.

${ }^{16}$ Newton wrote "I procured me a triangular glass prism to try therewith the celebrated phenomena of colours." From a letter Newton wrote to Henry Oldenberg, the Secretary of the Royal Society, 6 February 1672, quoted in full in Gallileo's Commandment: 2,500 Years of Great Scientific Writing, edited by Edmund Blair Bolles (Holt, New York, 2002), pp. 184188.

${ }^{17}$ Gerhard Herzberg, Atomic Spectra and Atomic Structure (Dover, New York, 1944).

${ }^{18}$ Hermann Haken and Hans Christoph Wolf, The Physics of Atoms and Quanta (Springer-Verlag, New York, 1990).

${ }^{19}$ Sune Svanberg, Atomic and Molecular Spectroscopy (Springer-Verlag, New York, 2004).

${ }^{20}$ Gerhard Herzberg, Molecular Spectra and Molecular Structure I: Spectra of Diatomic Molecules (Van Nostrand Reinhold, New York, 1950).

${ }^{21}$ Brian L. Sands, Morgan J. Welsh, Seda Kin, Ramesh Marhatta, Jacob D. Hinkle, and S. Burcin Bayram, "Raman scattering spectroscopy of liquid nitrogen molecules: An advanced undergraduate physics laboratory experiment," Am. J. Phys. 75, 488-495 (2007). (The cover photo for this issue contains a photograph that shows the visually stunning result of high-pulsed-power Raman scattering demonstrated in this laboratory.)

${ }^{22}$ Gerhard Herzberg, Molecular Spectra and Molecular Structure I: Spectra of Diatomic Molecules (Van Nostrand Reinhold, New York, 1950), pp. 193-204.

${ }^{23}$ Reference 22, p. 552. 